\title{
Part 8
}

\section{New theoretical developments in stellar pulsation and variability}




\title{
Theory of magneto-acoustic modes in roAp stars
}

\author{
Lionel Bigot \\ Niels Bohr Institute for Astronomy, Physics and Geophysics, Juliane \\ Maries Vej 30, DK-2100 Copenhagen $\emptyset$, Denmark
}

\begin{abstract}
In this review I present the effects of a strong magnetic field on the pulsations of rapidly oscillating Ap stars. I show how the field affects the observables, such as the frequencies and eigenvectors, and the selection of modes.
\end{abstract}

\section{Introduction}

The roAp (rapidly oscillating Ap) stars are a unique class of pulsators. These objects oscillate with short periods $(6-15 \mathrm{~min})$ and quite large amplitudes ( $\sim 1 \mathrm{mmag}$ ). Since their discovery two decades ago (Kurtz 1978), their number has grown up to 32 (2003 July). What make them unique is the role of the magnetic field in their oscillations. The field strengths in the Ap stars are in a range of a few hundred $\mathrm{G}$ up to a few $\mathrm{kG}$ and are the strongest magnetic fields on the main sequence.

A number of observational facts show us the important role played by the field in their oscillations. We may cite: the preference for dipole oscillations (i.e. the same geometry as the field; Kurtz 1982), the coincidence between magnetic and pulsation maxima (Kurtz et al. 1992) and more recently the detection of a variability of the magnetic field with the same period as that of the pulsation itself (Leone \& Kurtz 2003). More unusual observations are certainly related to the magnetic field: the unequal spacings of frequencies in the spectra of some multi-periodic roAp stars such as 10 Aql (Heller \& Kramer 1990) and HR 1217 (Kurtz et al. 1989, 2002) or the change in the shape of the light curve of the mode through the atmosphere of HR 3831 (Baldry \& Bedding 2000).

All these observations show that the strong magnetic field deeply modifies the properties of the oscillations. This then provides a great opportunity to make diagnostics of stellar magnetic fields using of the techniques of the asteroseismology. In this review, I present the influence of the strong magnetic field upon the properties of oscillations in roAp stars: frequencies, eigenvectors and damping.

\section{The theory of magnetic pulsations}

\subsection{The magnetic boundary layer}

The magnetic fields in roAp are typically of the order of a few $\mathrm{kG}$ (Hubrig et al. 2003), say two or three orders of magnitude larger than the global magnetic 


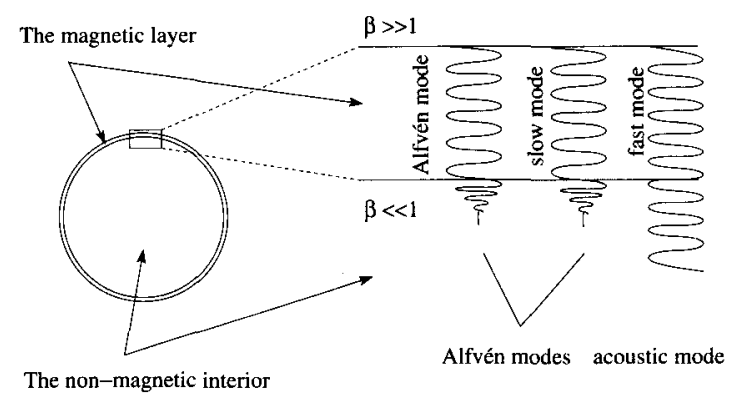

Figure 1. (Left) The star is divided into two zones. In most of the star, the mode is acoustic (where $\beta \ll 1$ ). Above, there is the thin magnetic layer where $\beta>1$. (Right) Zoom into the magnetic layer.

field of the Sun. However, even given that the field is so strong, the magnetic pressure remains negligible compared to the gas pressure in most of the star, except at the very surface in a thin layer that represents one to two percent in radius; see Fig. 1. In this magnetic layer the ratio $\beta$ between the magnetic pressure and the gas pressure is comparable to unity and even larger:

$$
\beta=\frac{P_{m a g}}{P_{g a s}}=\frac{B^{2}}{4 \pi P} \quad \sim 1 \quad \text { or } \quad>1 .
$$

The reason why the magnetic field has a such a strong influence on modes in roAp stars is due to a combination of two factors, a huge field strength at the surface together with a large amplitude of the mode in this magnetic layer. The latter condition is precisely fulfilled since the roAp stars pulsate with a high number of radial nodes $(n>20)$ which corresponds to large amplitudes in the outer layers $^{1}$. The consequence of this strong interaction between the mode and the magnetic field is a deep modification of the nature of the wave at the stellar surface which affects the global properties of the mode. For a realistic treatment of the magnetic effects, one must solve the linearized magneto-hydrodynamic (MHD) equations in this layer. Biront et al. (1982) were the first who proposed a treatment in this sense in the context of roAp stars.

\subsection{The magnetic waves}

In the presence of a magnetic field, the nature and number of waves in pulsating stars are different. The magnetic field modifies the restoring force of the existing waves and is responsible for a new type of wave, the so-called Alfvénic wave. This wave is a pure magnetic wave in the sense that its existence is only due to the presence of the magnetic field. Indeed, the restoring force is the tension created by the magnetic field lines. It therefore propagates only along the magnetic

\footnotetext{
${ }^{1}$ For comparison, the $\beta$ Cep stars, which are also magnetic pulsators, have magnetic fields somewhat similar (a few hundred $G$ ) to those of Ap stars, but the modes pulsate with very long periods (a few hours). The consequence is that the effects of the magnetic fields in these stars is by far much weaker than in roAp stars (3-4 orders of magnitudes lower).
} 
lines. The magnetic field modifies also the compressibility of the gas and thereby changes the restoring force of the acoustic modes that no longer exist. They are rather a mixed type between Alfvénic and acoustic waves and are called magneto-acoustic waves. A good understanding of what occurs in this magnetic layer can be achieved by a local analysis of the MHD system of equations. This is straightforward and can be found in many text books (e.g., Priest 1982). The phase velocities $v$ of the three types of waves are

$$
v_{ \pm}^{2}=\frac{1}{2}\left(c_{s}^{2}+v_{A}^{2} \pm \sqrt{\left(c_{s}^{2}+v_{A}^{2}\right)^{2}-4 v_{A}^{2} c_{s}^{2} \cos ^{2} \alpha}\right) \quad v=v_{A} \cos \alpha
$$

where $c_{s}=\sqrt{\Gamma_{1} P / \rho}$ is the sound speed, $v_{A}=B / \sqrt{4 \pi \rho}$ the Alfvénic speed and $\alpha$ the angle between the direction of propagation and the magnetic field. They are called fast and slow magneto-acoustic waves for obvious reasons. Note that we ignore for simplicity the contribution from gravity. In the magnetic layer $c_{s}$ and $v_{A}$ are comparable $(\beta \sim 1)$ which makes the phase velocities of the slow and fast modes also comparable. Both are then strongly interacting. However, below the magnetic layer $(\beta \rightarrow 0)$ we have: $v_{+} \rightarrow c_{s}\left(1+\frac{\beta}{2} \sin ^{2} \alpha\right)$ and $v_{-} \rightarrow v_{A} \cos \alpha$, which indicates that the two waves decouple $\left(v_{-} \ll v_{+}\right)$and evolve separately.

The picture of pulsations is represented in Fig. 1. The nature of the waves is rapidly changing through the outer layers of the star following the rapid increase of the gas pressure inward. In the magnetic layer there are three magnetic waves: one pure Alfvénic wave and two magneto-acoustic waves (slow and fast) strongly coupled. Deeper in the star, where the gas pressure is much larger than the magnetic one, they decouple and evolved independently. The fast mode transforms into an acoustic mode and the slow mode gives an Alfvénic wave.

\subsection{The Alfvénic damping}

Roberts \& Soward (1982), and later Campbell \& Papaloizou (1986), proposed an Alfvénic damping mechanism for magnetic pulsating stars. In most of the star (where $\beta \ll 1$ ) the mode pulsates like an acoustic mode. But at the surface this pulsation shakes and twists the magnetic field lines which produces Alfvénic waves. Their characteristic is a very short wavelength, much smaller than the pressure scale height below the magnetic layer, $\lambda_{A} / H_{p} \sim \beta$. This makes them very sensitive to dissipative processes (viscous, ohmic, radiative) and they are therefore rapidly damped when going into the interior of the star. This mechanism is a global damping for the acoustic mode. Indeed, during the pulsation cycle, an important fraction of the kinetic energy of the mode is used to produce these Alfvénic waves. The consequence is that the problem of oscillations in presence of a magnetic field is non-hermitian and the frequencies are complex. Depending how the mode oscillates across the magnetic field, it will generate more or fewer Alfvénic waves. Hence, this process is also an efficient selection mechanism for eigenmodes in magnetic stars.

\subsection{The distortion of acoustic mode}

Below the magnetic layer, the magnetic field has no direct effects $(\beta \ll 1)$ and the nature of the mode is purely acoustic. However, it is still affected by the surface magnetic layers which create a latitudinal dependence due to the 

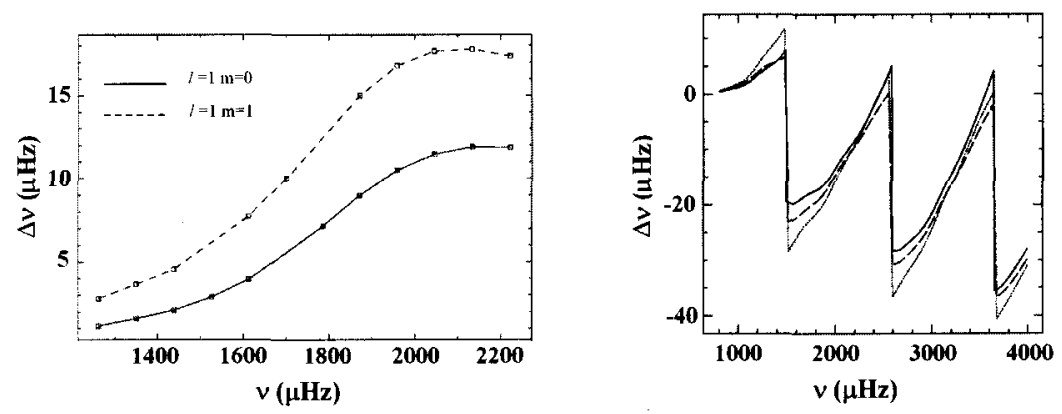

Figure 2. (Left) Magnetic shifts of the frequency for dipole modes as functions of the frequency; from Bigot et al. (2000); full lines $(m=0)$ and dashed lines $(|m|=1), B=0.8 \mathrm{kG}$. (Right) the same quantities for various $\ell$; from Cunha \& Gough (2000).

Lorentz force. The boundary condition for these modes is then non-spherically symmetric. This produces a distortion of the angular geometry of the mode. In practice, it means that each eigenmode is no longer described by a single spherical harmonic $Y_{\ell}^{m}$, as it is in the non-magnetic case, but rather by a linear combination of $Y_{\ell}^{m}$. The coupling occurs through the integral

$$
\int Y_{\ell^{\prime}}^{m^{\prime *}}\left(Y_{k}^{s}\right)^{2} Y_{\ell}^{m} d \cos \theta d \phi,
$$

where $Y_{k}^{s}$ is the spherical harmonic that corresponds to the magnetic field geometry. The square-dependence comes from the $B^{2}$ of the Lorentz force. The coupling exists as long as this integral does not vanish. In the case of a dipole magnetic field $(k=1, s=0)$, the effect appears as a Legendre polynomial $P_{2}$. The selection rule is then : $\ell+\ell^{\prime}=$ even and $m=m^{\prime}$. Hence there is no coupling between azimuthal orders $m$, but a coupling between modes with degrees of the same parity. The perturbed quantities are written as:

$$
\frac{\delta p}{p} \propto \sum_{\ell=m}\left(\frac{\delta p}{p}\right)_{\ell} Y_{\ell}^{m}
$$

\subsection{Raising the $(2 \ell+1)$ degeneracy of the mode}

In absence of a symmetry-breaking agent such as a magnetic field or rotation, the star is invariant by rotation. This means there is complete freedom in the orientation of the coordinate system which corresponds to a degeneracy of the perturbed quantities according to $m$. For a given degree $\ell$, all the modes $m=[-\ell, \ell]$ have the same frequency. The magnetic field breaks this symmetry and raises this degeneracy; see Fig. 2. This process is equivalent to the Zeeman effect in atomic physics. However, the magnetic field cannot make any difference between $+m$ and $-m$ modes. The modes remain then partially degenerate and the perturbed quantities depend only on $|m|$. 

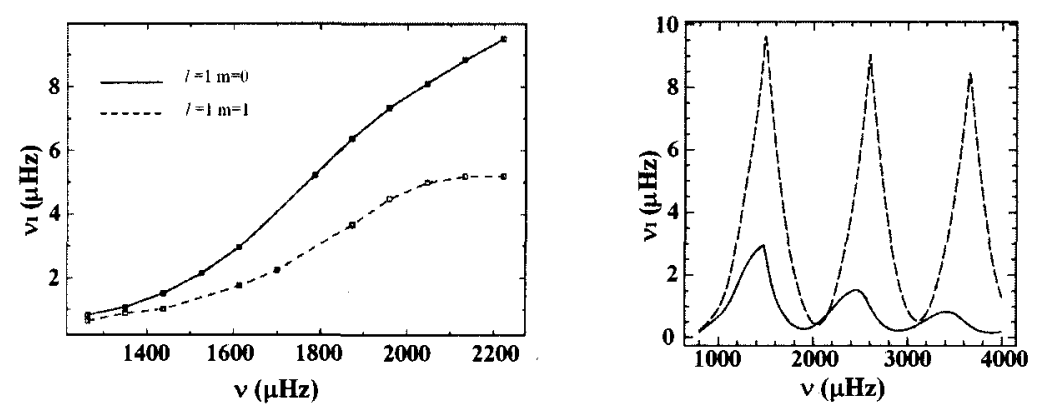

Figure 3. Same as Fig. 2, but for the imaginary part of the frequency. (Left) from B00: full lines $(m=0)$ and dashed lines $(|m|=1)$. (Right) from CG00: full lines $(m=1)$ and dashed lines $(m=0)$.

\section{Results of different models}

The consequences of the magnetic field interacting with the eigenmodes through the thin surface magnetic layer are very important for the asteroseismology of roAp stars. This affects all the observables associated with the pulsations: frequencies, eigenvectors and mode stability. In this section I compare numerical results obtained by the current models of pulsation in roAp stars. These models solve the MHD equations using non-perturbative approaches. They can be found in Dziembowski \& Goode (1996; DG96), Bigot et al. (2000; B00), Cunha \& Gough (2000; CG00) and Saio \& Gautschy (2002; SG00). The physical assumptions are the same between these models: ideal MHD, dipole magnetic field, adiabatic oscillations. What differs is the method used in solving MHD equations in the magnetic layer; see the references for more details.

\subsection{Shifts of frequencies - interpretation of spectra}

The magnetic field affects frequencies because it modifies the phase velocity of the mode and changes the path of the acoustic wave below the magnetic layer.

\subsection{Problems posed by the magnetic field.}

The value of the shift is of the order of a few $\mu \mathrm{Hz}$, typically $1-20 \mu \mathrm{Hz}$, for the range of magnetic strengths and frequencies found in roAp stars. This value is small compared with the absolute value of the frequency, typically $\nu=1000-3000 \mu \mathrm{Hz}$. However, what really matters for asteroseismology is the comparison of these shifts with the separations between consecutive eigenmodes in the frequency spectra. These shifts are then not negligible compared with the large separations, i.e. $\nu_{n, \ell}-\nu_{n-1, \ell}(\sim 30-80, \mu \mathrm{Hz}$, e.g. Shibahashi \& Saio 1985), and are equivalent to the small separations, i.e. $\nu_{n, \ell}-\nu_{n-1, \ell+2}$. The latter separations are very sensitive to the deepest layers of the star and are usually used to put some constraints on the core and its evolution. The shifts induced by the magnetic field mask this signature; see DG96 or B00. 

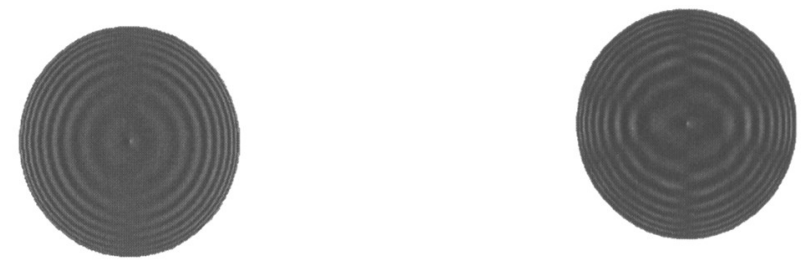

Figure 4. Radial dependence of a mode affected by a magnetic field. (Left) A dipole acoustic mode $(\mathrm{n}=23, \ell=1)$. (Right) The same mode affected by a dipole magnetic field of $0.7 \mathrm{kG}$.

\subsection{Comparisons between the results of the different pulsation mod- els.}

As it is clearly visible in Fig. 2, the behaviour of these shifts is very different from one model to the other. Indeed, in DG96 or B00 the shifts are smoothly increasing with frequency and/or magnetic field, whereas in CG00 or SG02 these shifts show some jumps that occur for some specific values of the frequency or the magnetic field. Another difference is the sign of the shift of frequency that may be negative in CG00 or SG02 and not in DG96 or B00. The reason for this different behaviour is still not known. The physical assumptions are the same, only the treatment is different. Saio (private communication) claimed that the source of difference may come from the fact that DG96 or B00 did not continue their calculations for magnetic fields or frequencies larger than those that they plot. SG02 used both polytropic and real stellar models for their calculations and found that the jumps occur for smaller frequencies/magnetic fields when polytropic models are used.

\subsection{Applications for two roAp stars: 10 Aql and HR 1217.}

A direct comparison between the observed spectra and numerical calculations has been done for two roAp stars: 10 Aql (Bigot \& Weiss 2002) and HR 1217 (Cunha 2001). In the first case, the three detected modes show an asymmetry in the frequency separations of about $5 \mu \mathrm{Hz}$ that cannot be explained neither by non-adiabatic effects or rotation, which would require unrealistic periods of rotation (too short). Since the magnetic field affects consecutive eigenmodes in a different way, it is a source of asymmetries in the frequency separations. Bigot \& Weiss (2002) show that a magnetic field of about $800 \mathrm{G}$ can reproduce such asymmetry well.

The problem of HR 1217 is more complex. In the data published by Kurtz et al. (1989) there are five consecutive eigenmodes $\left(f_{1}-f_{5}\right)$ almost equally spaced by about $33 \mu \mathrm{Hz}$, and one extra mode $\left(f_{6}\right)$ separated by 1.5 times this value. Such an observation cannot be reproduced by classical pulsation models. Cunha (2001) proposed an explanation based on the results of CG00. The mode that should be placed at $33 \mu \mathrm{Hz}$ from $f_{5}$ is missing because the Alfvénic losses are particularly strong for this frequency. The mode $f_{6}$ is shifted by about $-15 \mu \mathrm{Hz}$. Both propositions are compatible with the behaviour shown in fig. 3. Recently Kurtz et al. (2002) published new data for HR 1217 from a WET campaign. They clearly show that the "missing" mode is now present. If the explanation of 


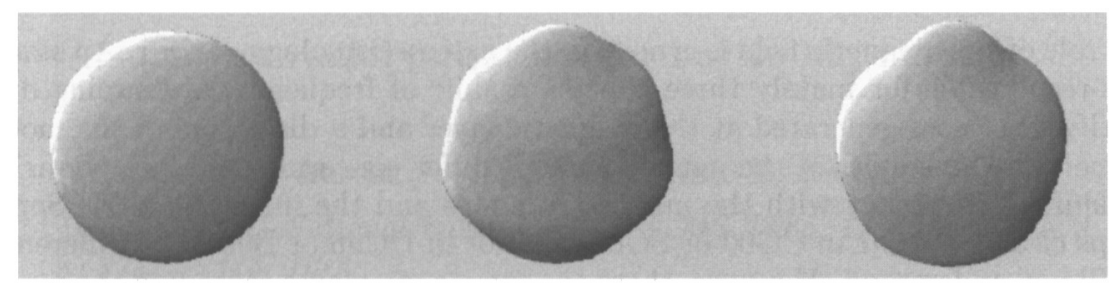

Figure 5. Evolution of the shape of a dipole mode by a change of the magnetic field. (Left) In absence of a magnetic field, $B=0 \mathrm{kG}$, the mode is a dipole $(\ell=1)$. It conserves the spherical symmetry of the star during the pulsation cycle. (Middle) The same mode with $B=0.7 \mathrm{kG}$. The mode is now a linear combination of degrees $\ell=1,3,5,7$. The surface is no longer spherical, but is vibrating under the influence of the components $\ell>1$. (Right) The same mode with $B=1.5 \mathrm{kG}$. The component $\ell=5$ of the mode dominates the others.

Alfvénic losses is correct, then the question is why this mechanism was efficient in 1989 and not in 2002. It is clear that the understanding of the pulsations in this star needs more theoretical and observational investigations.

\subsection{Damping of oscillations - mode selection}

In all studies of the magnetic effects on pulsations (DG96, B00, CG00, SG02) the damping due to Alfvénic losses has been found to be important. An example is given in Fig. 3. The imaginary part of the frequency is typically about a few $\mu \mathrm{Hz}$. In their two studies concerning non-axisymmetric modes $(m \neq 0)$ B00 and CG00 show different behaviour for the damping rates, but they agree that the non-axisymmetric modes are more stable against Alfvénic losses than the axisymmetric ones. This was unexpected since it was thought that $m=0$ modes, i.e. those aligned with the magnetic field, should be more favoured. In fact, they generate more Alfvénic waves at the magnetic poles where the field lines are vertical and feel then a more efficient dissipation.

\subsection{Effects on eigenvectors - mode identification}

The angular dependence of the mode is affected by the Lorentz force at the stellar surface. An example of the global radial distortion is given in Fig. 4. The shape of the mode is really affected even for relatively weak fields $(\sim 1 \mathrm{kG})$. What really matters for the observer is the aspect of the mode at the surface. This problem is illustrated in Fig. 5. I show a sequence of three modes corresponding to the evolution of a dipole mode by an increasing magnetic field. Its aspect is completely changed by the Lorentz force. One may easily appreciate the consequences in terms of the mode identification and the risk of misinterpreting the data. This example illustrates again the importance of taking into account the magnetic field in roAp stars. 


\section{Conclusions and perspectives}

The role of the magnetic field is crucial in the asteroseismology of the roAp stars. It is responsible for mainly three effects: a shift of frequency, a damping due to Alfvénic losses generated at the magnetic layer and a distortion of the mode geometry. The results of the current models differ concerning the behaviour of the shift of frequency with the number of nodes and the magnetic field. Some jumps clearly appear in CG00 or SG02 and not in DG96 or B00. The difference is still not understood. However, there are some agreements regarding the mode selection by the Alfvénic losses: the non-axisymmetric modes are more stable than the axisymmetric ones.

More realistic models are needed for a better comparison with observations. Indeed, the short wavelength of the mode at the surface makes these modes very sensitive to dissipative processes like resistive or radiative losses. None of the current models take these effects into account.

\section{References}

Baldry, I.K., Bedding, T.R. 2000, MNRAS, 318,341

Bigot, L., Provost, J., Berthomieu, G., Dziembowski, W.A., Goode, P.R. 2000, $\mathrm{A} \& \mathrm{~A}, 356,218[\mathrm{~B} 00]$

Bigot, L., Weiss, W.W. 2002, Communications in Asteroseismology, 141, 26

Bigot, L., Dziembowski, W.A. 2002, A\&A, 391, 235

Biront, D., Goossens, M., Cousens, A., Mestel, L. 1982, MNRAS, 201, 619

Campbell, C.G., Papaloizou, J.C.B. 1986, MNRAS, 220, 577

Cunha, M.S., Gough, D.O. 2000, MNRAS, 319, 1020 [CG00]

Cunha, M!S. 2001, MNRAS, 325, 373

Dziembowski, W.A., Goode, P.R. 1996, ApJ, 458, 338 [DG96]

Heller, C.H., Kramer, K.S. 1990, MNRAS, 244, 372

Hubrig, S., Kurtz, D.W., Bagnulo, S., Szeifert, T., Schoeller, M., Mathys, G., Dziembowski, W.A. 2003, A\&A, in press

Kurtz, D.W. 1978, IBVS, 1436, 1

Kurtz, D.W. 1982, MNRAS, 200, 807

Kurtz, D.W., Matthews, J.M., Martinez, P., et al. 1989, MNRAS, 240, 881

Kurtz, D.W., Kanaan, A., Martinez, P., Tripe, P. 1992, MNRAS, 255, 289

Kurtz, D.W., Kawaler, S.D., Riddle, R.L., et al. 2002, MNRAS, 330L, 57

Leone, F., Kurtz, D.W. 2003, A\&A, 407, 67L

Priest, E.R. 1982, Solar Magnetohydrodynamics, D. Reidel Publ., (Dordrecht)

Roberts, P.H., Soward, A.,M. 1983, MNRAS, 205,1171

Saio, H., Gautschy, A. 2002, in ASP Conf. Ser., Vol. 259, Radial and Nonradial Pulsations as Probes of Stellar Physics, eds. C. Aerts, T.R. Bedding, \& J. Christensen-Dalsgaard, (San Francisco: ASP), 294 [SG02]

Shibahashi, H., Saio, H. 1985, PASJ, 37, 245 